Res Publica. Revista de Historia de las Ideas Políticas

ISSN: $1576-4184$

\title{
Aprender desde las resistencias feministas en Latinoamérica: la lucha contra el capitalismo globalizado
}

\author{
Clara Navarro Ruiz*1
}

Recibido: 5 de junio de 2018 / Aceptado: 8 de octubre de 2018

Resumen. En los últimos años asistimos a un proceso de negociación de distintos Tratados de Comercio e Inversión (TTCI) que parecen amenazar nuestros derechos fundamentales. En las siguientes líneas abordamos este proceso y sus posibles riesgos. A continuación, gracias a la obra de Amaia Pérez Orozco, analizamos algunas de las posibles estrategias de resistencia a los mismos. Las experiencias feministas latinoamericanas en relación con el Tratado de Comercio ALCA, suspendido en 2005, son la base su argumentación.

Palabras clave: Tratados de Libre Comercio; Economía Feminista; Latinoamérica.

\section{[en] Learning from the South American Feminist Resistance: the Struggle against Globalized Capitalism}

\begin{abstract}
Within the last years, we are witnessing a process of negotiation of diverse Free-Trade and Investment Agreements (FTAs) which seem to threaten our fundamental rights. In the next lines we tackle this process and its possible risks. Following to that, and thanks to Amaia Perez Orozco's texts, we analyze some of the possible resistance strategies against them. The experience of South American feminists related to the ALCA Free-Trade Agreement, suspended in 2005, are the core of her argumentation.
\end{abstract}

Keywords: FTAs; Feminist Economics; South America.

Sumario: 1. Los tratados de Comercio e Inversión y el Poder Corporativo. 2. Resistencias feministas: el caso ALCA. 3. La mirada feminista ante los tratados de Comercio e Inversión.

Cómo citar: Navarro Ruiz, C. (2018). Aprender desde las resistencias feministas en Latinoamérica: la lucha contra el capitalismo globalizado, en Res publica 21.3, 545-557.

En las siguientes líneas vamos a realizar un análisis del texto Aprendizajes de las resistencias feministas latinoamericanas a los Tratados de Comercio e Inversión, de Amaia Pérez Orozco. Este texto se comprende como un puente entre lo teórico y lo

\footnotetext{
* Universidad Complutense de Madrid claranavarroruiz@gmail.com

1 Becaria Predoctoral en el Departamento de Filosofía y Sociedad de la Universidad Complutense de Madrid bajo la dirección de Pablo López Álvarez (Beca UCM-Santander). Aprovecho estas líneas para agradecer el encuentro de estos textos y la discusión sobre los mismos al Eje de Economía y Precariedad, sin quienes no hubiese sido posible saber tan siquiera de su existencia. Todos los errores de interpretación y olvidos que aquí haya son de cosecha propia.
} 
práctico, que intenta buscar alternativas de transformación y análisis de nuestro presente a partir de las experiencias acaecidas en Latinoamérica con el tratado ALCA, derrotado en la pasada década. Así, en primer lugar, daremos una explicación breve de en qué consisten los tratados de Comercio e Inversión. Los relacionaremos con el contexto en que éstos han de ser entendidos, que no es otro que la predominancia del poder corporativo a nivel global. En segundo lugar, describiremos el texto de Pérez Orozco, y, a partir de sus características y alguna otra referencia de la autora, se intentará ver qué puede significar tener una perspectiva feminista en el análisis de este tratado. Por último, y a la luz de lo analizado, se mencionará otro proyecto, el Tratado Internacional de los Pueblos, un texto de voluntad jurídica que también puede comprenderse como un resultado tanto de la hibridación entre teoría y práctica como de la relación entre Norte y Sur Global, concluyendo con esta consideración.

\section{Los tratados de Comercio e Inversión y el Poder Corporativo}

Los tratados de Comercio e Inversión (TCI) son convenios de carácter bi- o multilateral con un doble objetivo: buscan facilitar el comercio entre los países firmantes a través de la homogeneización de la legislación mercantil, la liberalización de mercados y sectores, entre otras medidas; al tiempo que garantizan las inversiones recíprocas que puedan darse en su relación financiera. Si bien este doble objetivo separó en etapas anteriores a los acuerdos en acuerdos de comercio por un lado, y acuerdos de inversión por otro, hoy se suelen acordar ambos objetivos en conjunto. Para que nos hagamos una idea de la importancia de los acuerdos en nuestro presente mundial, cabe destacar que a 15 de Diciembre de 2008 había 238 acuerdos comerciales de este tipo notificados a la Organización Mundial del Comercio (OMC), bien vigentes (como, ahora, el CETA), o en vías de negociación; mientras que hoy en día hay en torno a 3200 según la OIT $^{2}$. Muchos individuos son conscientes de que estos tratados suponen un instrumento que pone en riesgo los derechos adquiridos en materia medioambiental y laboral, pero sin saber exactamente por qué.

Para concretar algunas de las características que suelen tener estos tratados, quizá sea buena idea ver algunos de los rasgos que contenía el acuerdo previo del TTIP, el Tratado Internacional de Comercio e Inversión que actualmente se está negociando entre Europa y EE.UU. Digamos, de entrada, que las características que vamos a nombrar en ningún caso provienen de documentos publicados por la Comisión Europea o el Gobierno de EE.UU ${ }^{3}$. Es sabido que esta negociación está siendo llevada a cabo con un grado de secretismo intolerable, y las siguientes notas son fruto del trabajo de resumen de diversos teóricos y activistas que se han podido beneficiar de las filtraciones de Wikileaks o Greenpeace Holanda en 2014.

2 R. Moussié, Challenging corporate power. Struggles for women's rights, economic and gender justice. Toronto, Association for Women's Rights in Development (AWID) and Solidarity Center, 2016, p. 16.

3 Cabe destacar que los propios interlocutores en el proceso de negociación, al menos en el caso de Europa, no han sido directamente elegidos por los ciudadanos, lo que ya es muestra del pobre índice democrático de este acuerdo. Las presiones, argumentos y asesoramiento que puedan estar recibiendo tampoco proviene en su mayoría de entidades sociales: de 597 encuentros que ha tenido la Comisión Europea a este respecto, el 88\% ha sido con grupos de presión de carácter empresarial (Cf. A. Garay Zarra, Feministok TTIP-ARI Aurre! ¿Por qué es necesaria una respuesta feminista a los tratados globales?Bilbao, Mundubat, 2016, pp. 55). 
Lo que puede adivinarse en los documentos filtrados es que un tratado de este tipo supondría el asentamiento definitivo de la hegemonía de las fuerzas defensoras de los intereses capitalistas. Primero ${ }^{4}$, porque se propone una liberalización de los servicios y la contratación pública, aplicando a las empresas extranjeras que deseen prestar servicios los principios legislativos nacionales. Además, la lista de excepciones a este punto es meramente negativa, por lo que no se incluyen las prestaciones aún no existentes. En segundo lugar, porque se da una eliminación de las barreras de acceso al mercado. Esto se traduce en una erradicación de las limitaciones no arancelarias, es decir, de dispositivos de control y administración de carácter público. Cabe destacar que una convergencia en términos regulatorios es especialmente grave si tenemos en cuenta que EE.UU. no ha firmado 6 de los 8 Convenios Fundamentales de la OIT, ni reconoce la Convención de las NN.UU. sobre la Eliminación de todas las formas de Discriminación contra la Mujer (CEDAW). Por último, y por lo que respecta a la protección de las inversiones, el punto más polémico ha sido la instauración de un sistema de arbitraje privado para la solución de las posibles disputas entre naciones y las empresas multinacionales. El Investor-State-Dispute-Settlement (ISDS) proponía un sistema en que, sin pasar por las vías judiciales del país en cuestión, decidiera sobre el conflicto. Este instrumento era de disfrute unilateral por parte de las empresas extranjeras, y los árbitros no eran jueces, sino abogados en tribunales constituidos ad hoc, cuya imparcialidad sería más que discutible. Si bien, tras la filtración, este último organismo ha sido eliminado en versión de otro instrumento (ICS) que incluye algunas mejoras procesales, sus aspectos más problemáticos no han sido solventados ${ }^{5}$. Basándonos en las características del TTIP, es evidente que los tratados de comercio e inversión tienen un fuerte impacto en la capacidad de reacción de los Estados-nación, en tanto instaura una relación de asimetría de estos con respecto a los agentes económicos, así como en la protección de los derechos más básicos: la homogeneización legislativa que busca mejorar y garantizar los índices macroeconómicos se realiza siempre a costa de los trabajadorxs y el planeta.

Incluso a pesar de estos presagios, desde la opinión pública se comenta cuan necesario y beneficiosa es la apertura de los mercados al extranjero, ya que cualquier otro tipo de política que tenga como vía algún tipo de proteccionismo sólo puede desembocar en una guerra comercial, de la que los medios nos alertan con insistencia. La experiencia latinoamericana nos puede ayudar aquí a saber si esas promesas realizadas desde los poderes públicos alcanzan un nivel de verosimilitud aceptable, al menos en lo que respecta en términos de desigualdad de género. Precisamente, y durante la década pasada, el Capítulo Latinoamericano de la Red de Género y Comercio se encargó de realizar un análisis en múltiples países (Argentina, Chile, Uruguay, Colombia, etc.) acerca de la incidencia de la liberalización en este aspecto ${ }^{7}$. Se analizaron el empleo, los servicios estructurales y la economía del cuidado. El

\footnotetext{
Cf. Ibidem, pp. 46 y ss.

Cf. P. Eberhardt, El ISDS zombi. Renombrado como ICS, el derecho a demandar de las multinacionales de demandar a los Estados se niega a morir, publicado por Publicado por Corporate Europe Observatory (CEO), Association Internationale de Techniciens, Experts et Chercheurs (AITEC), Attac Austria, Campact, ClientEarth, Ecologistas en acción, Forum Umwelt \& Entwicklung, et al., 2016. [Disponible en: https://www.ecologistasenaccion.org/wp-content/uploads/adjuntos-spip/pdf/isds-zombi.pdf].

6 Cf. A. Garay Zarra, op. cit., pp. 16 y ss.

7 N. Bidegaín Ponte, Comercio y Desarrollo en América Latina: el orden de los factores afecta al producto. CIEDUR/IGTN-LA, 2008.
} 
resultado habla por sí solo. La reestructuración de los aparatos productivos en relación al comercio exterior puede ejemplificarse en la extensión y favorecimiento del llamado "modelo maquila", aquel que conjuga la pobreza salarial, la desprotección en términos de los seguros sociales de las trabajadoras, el fortalecimiento de formas informales de trabajo (desde el hogar, cobro por pieza), un fuerte impacto en las lógicas de reproducción y tiempos de cuidado, y un empleo alienante cuya cualificación no tiene carácter progresivo. Un ejemplo concreto de cómo los tratados de libre comercio ayudan a favorecer estas condiciones lo encontramos en el ejemplo del DR-CAFTA, el tratado que vincula a EE. UU. Con algunos países centroamericanos. En el Salvador, así, en el período 1993-2005 las exportaciones de la maquila habían aumentado de 288 a 1814 millones. En 2015, DR-CAFTA mediante, fueron de 2552 millones, un $46 \%$ de las exportaciones totales del país. Un fenómeno ligado a la llamada feminización de la pobreza, en que el género y la raza son recursos explícitos de las fuerzas capitalistas para mantener el nivel salarial al mínimo ${ }^{8}$.

Cabe ahora preguntarse sobre las características del poder que perpetúa y asientan estos tratados. Como ya se ha adelantado, sólo cabe calificarlo como un recurso del capitalismo global, que desde la irrupción de la crisis en 2008 se encuentra en un estado de permanente impasse que se busca solventar a toda costa. Ahora bien, este fenómeno no puede verse como un accidente o interrupción del buen funcionamiento del capitalismo. Al contrario, ha de verse como la irrupción a la superficie de una crisis estructural del sistema capitalista que comenzó en los años 70 , que pone de relieve que habríamos llegado al limite interno ${ }^{9}$ de la posibilidad de reproducción sin fricciones de nuestro sistema socioeconómico. Esta situación tiene su origen en la tercera revolución microelectrónica o tercera revolución industrial, que crea la posibilidad de creación de grandes masas de valor con una mínima incorporación de trabajo abstracto humano en el proceso de producción, a causa de la sofisticación actual de la maquinaria. Tras el agotamiento de los mecanismos de compensación propios del plusvalor relativo (expansión territorial el capital, abaratamiento de las mercancías, etc.), y la ruptura definitiva del aparente sueño dorado de la creación ficticia de riqueza en el mercado financiero (burbujas, crisis de las punto com), nos encontramos ante la imposibilidad del capital de valorizar el valor, es decir, de cumplir con la reproducción hasta el infinito del esquema Capital-Mercancía-Capital ampliado $\left(\mathrm{C}-\mathrm{M}-\mathrm{C}^{1}\right)$ garantizando al mismo tiempo el bienestar relativo de una masa de población suficiente para el mantenimiento de la paz social en los enclaves tradicionalmente favorecidos por la división mundial del trabajo.

Este conjunto de factores permiten afirmar, en definitiva, el agotamiento del sistema, sin que esto signifique que este vaya a deternerse de manera súbita. Antes bien, el concepto de "límite" y "colapso" han de concebirse como claves de lectura para la comprensión de nuestra época.

8 Cf. R. Moussié, op. cit. Es también interesante mencionar aquí el incisivo análisis que V. Chibber realiza de las fuerzas que someten al planeta entero en Postcolonial theory and the Specter of Capitalism, Londres, Verso Books, 2013.

9 La noción de "limite interno" ha sido desarrollada por los criticos de la escisión del valor. Para más información puede verse R. Kurz, Geld ohne Wert, Berlin, Horlemann, 2012, entre otros textos de este autor o los escritos de R. Scholz. Para una introducción a este concepto en lengua castellana puede verse C. Navarro Ruiz, "Fin de partida. Acerca de la noción de "límite interno" según la crítica de la escisión del valor", en Oxímora. Revista Internacional de Ideas Politicas, n9, "Crítica inmanente del capitalismo”, Otoño 2016. pp. 1-24. 
Algunas de las características de esta etapa ${ }^{10}$ son el sobredimensionamiento del sector financiero, la constante transferencia de recursos públicos al ámbito privado (en forma de ayuda y/o mecanismos de concertación público/privado), la terciarización de las sociedades occidentales y la acentuación del trabajo precario a través del fenómenos como el trabajo autónomo, la externalización y subcontratación; una política migratoria y de fronteras crecientemente excluyente y agresiva, una fiscalidad poco progresiva, un aumento de la violencia sobre los cuerpos de mujeres y los racializados, el impacto sobre el trabajo reproductivo no remunerado, etc.

Al mismo tiempo, esta explicación general se puede concretar si identificamos al mayor triunfador en este continuo proceso de reestructuración a nivel mundial: el poder corporativo. Este se caracteriza primordialmente por una considerable concentración de capital en sus manos, y un control excesivo sobre la administración y acceso a todos los ámbitos socioeconómicos de relevancia, como los recursos naturales, trabajo, información, finanzas, etc ${ }^{11}$. Un Su peso en la economía global actual es más que considerable, tal y como muestran datos como que el $63 \%$ de las entidades globales económicas sean multinacionales; o el hecho de que los beneficios de las tres corporaciones más grandes del mundo (Royal Dutch Shell, Exxon Mobil, Wal-Mart) fueran en 2012 según datos de la Oxfam mayores que el producto interior bruto (PIB) de 110 países, siendo el 55\% de ellos Estados-nación ${ }^{12}$.

La bases de la consolidación de este poder se da a través de una doble alianza entre corporaciones y/o empresas transnacionales y los gobiernos a escala mundial, y una "arquitectura jurídica de la impunidad"13 que ha permitido un creciente desequilibrio entre el estatus de obligatoriedad de los derechos comerciales respecto a los laborales y humanos a nivel internacional ${ }^{14}$. Este desequilibrio ha permitido el surgimiento del Derecho Corporativo Global o nueva lex mercatoria, con el que

las grandes corporaciones tutelan sus derechos, mientras no existen contrapesos suficientes ni mecanismos reales para el control de sus impactos sociales, laborales, culturales y ambientales [...]. Los derechos de las empresas transnacionales se blindan gracias a un ordenamiento jurídico global basado en reglas de comercio e inversiones cuyas características son imperativas, coercitivas y ejecutivas, a la vez que sus obligaciones se remiten a ordenamientos nacionales sometidos a la lógica neoliberal y un Derecho Internacional de los Derechos Humanos manifiestamente frágil. En este contexto surgen la Responsabilidad Social Corporativa (RSC) y los códigos de conducta voluntarios, unilaterales y sin exigibilidad jurídica, que

10 Respecto a esto, cf. R. Kurz, Das Weltkapital. Globalisierung und innere Schranken des modernen warenproduzierenden Systems. Berlín, Tiamat., 2005; A. Pérez Orozco, Subversión feminista de la economía. Aportes para un debate sobre el conflicto capital-vida. Madrid, Traficantes de Sueños, 2014 (En adelante Subversión...); C. Vela, Capitalismo terminal. Anotaciones a la sociedad implosiva. Madrid, Traficantes de Sueños, 2018.

11 Cf. R. Moussié, op. cit., p. 8.

12 Cf. Ibidem, p. 11.

13 A. Pérez Orozco, Aprendizajes de las resistencias feministas latinoamericanas a los tratados de comercio e inversión. Del no al ALCA al cuestionamiento del capitalismo patriarcal. Paz con Dignidad, OMAL, 2017, pp. 11. (En adelante Aprendizajes...).

14 Cf. J. Hernández Zubizarreta, "La responsabilidad social corporativa y las empresas transnacionales: de la ética de empresa a las relaciones de poder" en Lan Harremanak, 19, 2008-II, pp. 17-49, (en adelante: "La responsabilidad social corporativa...") y de este mismo autor, Lex Mercatoria, Definición disponible en OMAL. Observatorio de Multinacionales en América Latina [http://omal.info/spip.php?article4803], 2012. 
no son sino una forma de Derecho blando (soft law) como fórmulas alternativas o cualquier control jurídico ${ }^{15}$.

...es decir, utilizando en su beneficio todos los instrumentos jurídicos disponibles para la consolidación de sus intereses, siendo la lex mercatoria el instrumento para ello. Quizá sea pertinente detenerse brevemente en explicar más ampliamente esta nueva arquitectura jurídica ${ }^{16}$. El origen de esta situación puede achacarse a dos factores. En primer lugar, una nueva comprensión de la empresa a partir de los años 80, que se suponía más comprometida con la sociedad en su conjunto, así como más creativa y flexible en sus ordenamientos internos. Se comienzan entonces a vincular el compromiso con los Derechos Humanos o el respeto al Medio Ambiente con los objetivos sociales de la empresa, lo que da lugar al desarrollo de la disciplina de la Responsabilidad Social Corporativa (RSC), que a partir de los años 90 se añadirá a los estudios universitarios como materia de estudio. En segundo lugar y más principalmente, esta nueva arquitectura jurídica se debe a una contraofensiva del capital desde la Cámara de Comercio Internacional, que ocurrió en los años 70. En esta década, los capitales transnacionales empezaban a despuntar, y desde las Naciones Unidas se planteaba entonces la introducción de un código externo vinculante para el control de su poder, que controlara su capacidad de intervención por medio de la subordinación a intereses generales. En este debate estuvieron presentes figuras como la Salvador Allende ${ }^{17}$. La respuesta de los empresarios no se hizo esperar, y a lo largo de esta misma década aprobaron más de 300 códigos empresariales propios que hicieran obsoleto el primero. Con ello consiguieron asimismo desplazar el debate de la voluntariedad u obligatoriedad de las normativas a organismos donde su capacidad de influencia fuera mayor, como la OCDE o la OIT ${ }^{18}$.

El conjunto de ambos factores ha desembocado en el hecho de que las obligaciones laborales de las empresas transnacionales se vertebren únicamente a través de mecanismos del Derecho blando (soft law), como una falsa alternativa al control jurídico. Esta categoría se vincula en el ámbito del Derecho Internacional con la necesidad de dotar de cierta utilidad a normas que los Estados adoptan en la teoría, pero que no cumplen o pueden cumplir en la práctica. Es a causa de dicho motivo

15 J. Hernández, E. González, P. Ramiro, “Tratado Internacional de los pueblos para el control de las empresas transnacionales. Una apuesta desde los movimientos sociales y la solidaridad internacional". En Cuadernos de Trabajo Hegoa, $\mathrm{n}^{\circ} 64,2014$, p. 7. Instituto de Estudios sobre Desarrollo y Cooperación Internacional, Universidad del País Vasco.

16 Esta nueva arquitectura jurídica es lo que el análisis de N. Brenner, J. Peck y N. Theodore identifica claramente con el neoliberalismo. Para ellos el neoliberalismo es "una tendencia históricamente específica, pautada, híbrida y desarrollada desigualmente de estructuración regulatoria sujeta a la disciplina de mercado" (24) que ha de analizarse en base a tres elementos: los experimentos regulatorios que se realizan en los distintos territorios para intensificar la disciplina mercantil, así como el análisis de sistemas de transferencia normativa interjurisdiccional, es decir, el análisis de las estructuras que permiten la expansión de las políticas neoliberales (organismos, instituciones, etc.). Por último han de considerarse los regímenes normativos transnacionales a gran escala, como los TCI. (V. N. Brenner, J. Peck y N. Theodore "¿Y después de la neoliberalización? Estrategias metodológicas para la investigación de las transformaciones regulatorias contemporáneas", en Urban, 1, 2010: pp. 21-40, así como C. Navarro Ruiz, "Prolegómenos a una economía alternativa: el neoliberalismo como racionalidad política", en Pensamiento al margen, nº, 2016, "Políticas económicas alternativas al neoliberalismo", pp. 25-52.

17 Cf. J. Hernández, "La responsabilidad social corporativa...", pp. 40 y ss.

18 A este respecto, resulta muy iluminadora la actitud de la representación de los empresarios en la OIT en lo referente a la introducción de convenios internacionales sobre un asunto tan de actualidad como la subcontratación. Se han negado sistemáticamente a traducir en exigibilidad jurídica lo que ellos promulgan en el plano de la responsabilidad ética. (Cf. ibidem). 
que este tipo de normas adquieren entonces un cierto estatus de deseo, que se espera pueda influir posteriormente en la elaboración de normativas.

En el caso de las empresas transnacionales, esta noción se ha convertido, sin duda alguna, en un instrumento para el asentamiento de la unilateralidad y la voluntariedad en el compromiso la autorregulación de sus conductas. Se espera de las propias empresas capitalistas que autoadministren el cumplimiento de sus compromisos éticos respecto al cumplimiento de los derechos laborales y humanos de sus actividades. Teniendo en cuenta que el único fin de la empresa capitalista es la obtención del beneficio a costa de la permanente extracción de plusvalor de sus trabajadores, esta medida supone algo así como esperar autocontrol de un pirómano al que se regala diariamente una caja de cerillas. Sea como fuere, esta interpretación y utilización del Derecho blando ya está fuertemente instalada, tal y como muestra Global Compact, una iniciativa de las Naciones Unidas creada en 2011, de carácter voluntario. Ésta invita y apoya a las grandes empresas al cumplimiento de 10 compromisos en materia de DD.HH., trabajo, medio ambiente y lucha contra la corrupción ${ }^{19}$.

Este es, a muy grandes rasgos, el panorama al que nos enfrentamos tanto el Norte como en el Sur global, que sin duda alguna ha de calificarse, cuanto menos, de preocupante. Ahora bien, sólo tiene carácter de novedad en una parte del globo, la Occidental, dado que el Sur lleva desde el anterior siglo enfrentándose a estas lógicas que sólo tras la crisis hemos empezado a experimentar. Pioneros en la desgracia del sufrimiento generado por estas políticas, pero también en las formas de resistencia a las mismas. Particularmente exitoso fue la derogación del ALCA, el Tratado de Comercio entre EE. UU., Canadá y América Latina, que se llevó finalmente a cabo en 2005. Esta experiencia es la que, en lo que resta, vamos a analizar, en busca de perspectivas que puedan ayudarnos a analizar y transformar nuestro presente.

\section{Resistencias feministas: el caso ALCA}

El Libre Tratado de Comercio de las Américas (ALCA) se proyectó en 1994 en la ciudad de Miami, como un acuerdo que buscaba disminuir al máximo las barreras arancelarias entre EE. UU., y la mayoría de países latinoamericanos, exceptuando Cuba. El proyecto ${ }^{20}$ partía de una antigua ambición de dominio comercial de los Estados Unidos sobre la región de Abya Yala ${ }^{21}$, una estrategia en continuidad con los Programas de Ajuste Estructural (PAE) que se habían ido implementando en toda la región a partir de los años 80 . La gran oposición que sufrió desde un primer momento, orquestada por un conjunto heterogéneo de movimientos sociales de diversas procedencias (movimientos sociales, feministas, medioambientales, indigenistas, etc.), provocó su derogación implícita en la IV Cumbre de las Américas celebrada en 2005, donde Estados Unidos no pudo imponer en el documento final el compromiso por la reapertura de las negociaciones en el documento de conclusiones del encuentro.

El texto de Amaia Pérez Orozco, Aprendizajes de las resistencias feministas latinoamericanas a los tratados de comercio e inversión, es un documento elaborado

19 Puede consultarse su propia página web en el enlace [https://www.unglobalcompact.org/what-is-gc/mission/ principles].

20 Cf. A. Pérez Orozco, Aprendizajes..., p. 13.

21 Este se trata del nombre originario de América antes de su proceso de conquista imperial, que se utiliza en muchos contextos activistas como forma de ejercicio de memoria crítica. 
a través del análisis teórico de los fundamentos de los tratados de comercio y la situación específica de la región, además de la condensación de los aprendizajes que la autora ha adquirido por medio de entrevistas con diversas feministas que estuvieron al frente de la movilización. El resultado son una serie de pequeñas claves que pueden servir para leer con más fuerza el presente, desde una mirada feminista y anticapitalista.

Antes de comenzar con este texto, cabe preguntarse por qué se escoge aquí una mirada feminista, por encima de otras opciones, y particularmente, en qué se materializa dicha mirada en un contexto de crítica capitalista. De entrada, y como anécdota, el/ellx lectorx debería cuestionarse si tal pregunta hubiese aparecido como algo necesariamente superfluo si, en lugar de haber escogido una perspectiva feminista, se hubiese escogido una atenta únicamente a las relaciones entre capital y salario, algo que quien escribe estas líneas también se plantea. Nuestra decisión, en cualquier caso, parte de la propia convicción de que la mirada feminista a la economía y al sistema capitalista es la más completa, incisiva y crítica. Además proporciona una perspectiva de transformación que busca la emancipación de las relaciones sociales capitalistas en términos más concretos que los que se han dado tradicionalmente, en que una situación postcapitalista se relegó bien a un supuesto paraíso de la perfecta administración de recursos y capacidades ("de cada cual según sus capacidades, a cada cual según sus necesidades"); o bien al resultado de un proceso de desarrollo de un capitalismo de Estado (socialismo real) que en realidad, siguió siempre anclado a las formas sociales que quería superar (productivismo, desarrollismo, carácter biocida, etc.).

De esta manera, la economía feminista ${ }^{22}$ puede definirse como aquella mirada al sistema socioeconómico que busca comprenderlo y cuestionarlo a través del conflicto fundamental capital-vida, buscando su superación. Al contrario que la economía actual, que tiene como centro la valorización del valor de los capitales, lo que en la economía feminista está en el centro, y ha de guiar nuestras reflexiones, es la sostenibilidad de la vida. Esto implica ${ }^{23}$ tres cuestiones, a saber: no separar la economía de la sociedad y la política, poner la mirada en los centros donde se resuelve la vida (los hogares) y "observar la forma diferencial en que se encarna la economía". Aunque el sistema capitalista imponga la dictadura de la productividad y la explotación a nivel global, creando un sistema de clases según nuestra posición de acceso a la riqueza monetaria, no puede decirse que éste nos afecte a todxs del mismo modo, pues esto es algo que depende también del cuerpo que habitamos (raza, género, neurodiversidad, condiciones físicas, edad, etc.). Las siguientes palabras del colectivo

22 Obviamos aquí los planteamientos de corte neoliberal que buscan hacer ver el impacto de las relaciones sociales en la relación entre géneros, que, más que Economía Feminista, podría llamarse "Economía ortodoxa con cierta perspectiva de género". Asimismo, obviamos también los planteamientos que, si bien no necesariamente neoliberales, buscan el mantenimiento o mejora del sistema capitalista, y no necesariamente su transformación. (Para una diferenciación entre estas corrientes, así como una perspectiva crítica acerca de ellas, puede verse Amaia Pérez Orozco, Subversión.... Otros textos útiles para situarse en la relación del género la economía y el surgimiento de distintas posturas son C. Carrasco (Ed.), Mujeres y economía. Nuevas perspectivas para viejos y nuevos problemas. Barcelona, Icaria, 1999; así como C. Carrasco, C. Borderías y T. Torns (eds.), El trabajo de cuidados. Historia, teoría y politicas. Madrid, Los libros de la catarata, 2011.

23 Eje de Precariedad y Economía Feminista, "Eje de Precariedad y Economía Feminista; construcción colectiva del pensamiento y herramientas para transformas los espacios que habitamos", en VV. AA., Economía Feminista. Una alternativa al capitalismo, (en adelante Economía Feminista...) Bilbao, Mundubat, 2017, pp. 47-64, aquí p. 51. 
Herrialdes son útiles para situar esta perspectiva, ayudando a resolver las dudas que permanecieran sin resolver:

La economía feminista se ha venido demostrando como una herramienta imprescindible para abordar el conflicto capital-vida, a partir de las premisas que la fundamentan: reconocer la economía como algo que va más allá de las fronteras de esa economía clásica que la limita a su aplicación para el mercado lucrativo, incorporando el trabajo de cuidados no asalariado como parte del circuito económico; segunda "descubrir" el trabajo de cuidados y la consideración de que para comprender las relaciones de género (división sexual del trabajo) es clave entender el funcionamiento del sistema económico; y tercera [,] la economía feminista lleva implícito un compromiso político para contribuir a la construcción de un sistema socioeconómico más justo ${ }^{24}$.

Habida cuenta de estas cuestiones introductorias, Amaia Pérez Orozco identifica en la resistencia al ALCA diferentes factores, concretamente: tres aprendizajes necesarios, tres tensiones a tener en cuenta, y dos claves para el cuestionamiento del presente, desembocando así en la elaboración de los materiales para una perspectiva feminista de los TCI. Vayamos por orden.

Por lo que respecta a los aprendizajes ${ }^{25}$, las experiencias de las feministas latinoamericanas nos proponen criticar sin medias tintas y pasar del análisis del mero impacto a la denuncia de conjunto. El análisis de impacto, que, basándose en la lectura y análisis del propio tratado, busca hacer ver las consecuencias de lo que se pacta en una economía/s y o sociedad/es determinada/s que se presuponen de manera más o menos estática, puede ser útil. Sirve para llamar a la movilización y crear una posición común, que muy probablemente sea calificada de catastrofista por las instancias que buscan su oposición. Sin arredrarse ante tales acusaciones ha de irse a una comprensión global que permita entender el Tratado como parte de un desarrollo de fuerzas internas de carácter político, y que posibilite con ello desarrollar un tipo de crítica a instancias más globales que las fronteras de un solo Estado-nación. Sólo de esta manera se garantiza una oposición cada vez más robusta y mayoritaria, que apunte al problema más fundamental: el sistema capitalista como un modelo de civilización de carácter biocida. En segundo lugar, no se puede obviar el carácter heteropatriarcal del sistema que se está poniendo en cuestión. En consonancia con lo que antes se ha dicho acerca de la perspectiva de la economía feminista, ésta no puede ser obviada en nuestros análisis críticos: la división social del trabajo y la invisibilización del trabajo reproductivo fundamenta el neoliberalismo, asentado en los cuerpos y vidas de las mujeres. Esto ha de tener consecuencias necesarias tanto en las propuestas como en los procedimientos de movilización, incluso si ello hace que sean más dificultosos, como ocurrió en la oposición al $\mathrm{ALCA}^{26}$. En tercer lugar ${ }^{27}$, y como consecuencia de

24 C. Carrasco Bengoa y C. Díaz Corral, Economía feminista. Desafios, propuestas, alianzas. Barcelona, Entrepueblos/Entrepoubles/Entrepobes/Herriarte, 2017, p. 10.

25 A lo largo de estas páginas cf. A. Pérez Orozco, Aprendizajes..., pp. 24 y ss.

26 Pérez Orozco comenta que, si bien en la oposición al ALCA se asumió desde un principio la necesidad de la presencia de mujeres en todos los espacios políticos y la creación de criterios específicos asociados al género, también hubo dificultades en la inclusión de cláusulas provenientes de estos ámbitos, tanto en los propios tratados de libre comercio, como en el seno de los propios movimientos sociales (Cf. Ibidem, p. 36).

27 Cf. Ibidem, pp. 42 y ss. 
este último punto, hemos de beneficiarnos del punto de vista de las mujeres, que se encuentran en el centro de la economía - puesto que están presentes en los ámbitos de la producción y reproducción como sujetos activos, en conexión también con el acceso directo a los recursos naturales- para subordinar la economía a la realidad de la vida misma. Puesto que se parte de esta realidad concreta, la visión desde la vida de las mujeres nos permite asimismo politizar lo cotidiano y ampliar los frentes de resistencia, que también han de incluir iniciativas de autocuidado ${ }^{28}$.

Lo siguiente de lo que se hace cargo Pérez Orozco son las tensiones ${ }^{29}$, es decir, aquellas dinámicas generadas por la interacción de las distintas instancias, actores del proceso de movilización y decisiones rectoras (gobiernos, instituciones de cooperación, pero también ámbitos teóricos académicos o comités de expertos, establecimiento de prioridades, etc.) que pueden ser perjudiciales para el fortalecimiento de los movimientos sociales de oposición. La primera que se analiza es la necesidad de una cierta autonomía con respecto a las instancias gubernamentales a todos los niveles. El interés ha de ponerse en la necesidad salvaguardar dos factores fundamentales, la capacidad de crear agenda y la supresión del peligro del parcelamiento. Evidentemente, esto no excluye la colaboración de las primeras, pero se han de tener presentes los riesgos para la elaboración de mecanismos de compensación y prevención: participación únicamente intermitente y/o temporal, atención a los procedimientos de participación, etc. En segundo lugar ha de mencionarse la tensión que existe entre el conocimiento y análisis experto y la movilización política. Aunque el análisis de detalle de carácter técnico no puede ser obliterado, puesto que éste nos carga de razones, ha de alertarse sobre ciertos peligros. La posibilidad de creación de dos niveles de la movilización, casi al modo del antiguo cliché del marxismo tradicional (vanguardia teórica/masa proletaria) es quizá la más peligrosa. Invisibiliza la creación de conocimiento en otros niveles y puede hacernos caer en un excesivo pesimismo de la razón, que bloquee el ínsito optimismo de la voluntad. Los resultados más fructíferos en esta colaboración en el caso del ALCA fueron aquellos que promovieron estrategias de combinación. En estas, lo técnico se comprende como una herramienta al servicio de la movilización y se deja vertebrar por ella incluso en sus propias investigaciones, dejando que sea esta última donde se definan el discurso y la estrategia, planteando las preguntas de la teoría.

Una última tensión se encuentra en la necesidad de dejar de lado los planteamientos sectoriales que parcelan a los movimientos sociales. Aquí fue donde la oposición al ALCA se hizo robusta, en la construcción de una agenda común, tarea que no se realizó sin dificultades, puesto que el riesgo de fragmentación se encuentra tanto a nivel grupal, sectorial, como temático. En nuestra opinión, si bien esta problemática ha de ser tenida en cuenta, no hay manera de prevenirla, puesto que su superación sólo puede ser el resultado del propio proceso de discusión de las diversas posturas, $\mathrm{y}$, por lo tanto, habrá de repetirse a cada nueva movilización.

El texto comienza a cerrarse con la propuesta de dos claves para pensar nuestro presente. La primera de ellas es la rearticulación del neoliberalismo con la violencia patriarcal. Durante algún tiempo, pareció posible una articulación armoniosa entre las demandas de los derechos sexuales y reproductivos con las lógicas económicas capi-

28 Cf. A. M. Ferrera Chávez, "La economía feminista: una mirada desde la sostenibilidad de la vida", en VV. AA. Economía feminista... pp. 65-78.

29 Cf. A. Pérez Orozco, pp. 50- 72. 
talistas, algo que el aumento de la violencia en Colombia o los ataques machistas a D. Rousseff - también, por qué no decirlo, a Hillary Clinton en el Norte hegemónico- han desmentido. La violencia patriarcal ha de comenzar a considerarse un instrumento de control con efectos simbólicos, que se utiliza para expresar al conjunto de la sociedad los riesgos que se asumen al no atenerse a las demandas e intereses de los poderes fácticos. Así, la agresividad renovada del patriarcado es "una violencia expresiva, envía un mensaje: no es sólo mercantilizar la vida, sino de dominarla. Quien ejerce esa violencia, domina la vida" ${ }^{30}$. En este sentido, al movimiento feminista, según Pérez Orozco, le corresponde un papel político determinante, consistente en la comprensión del papel estructural de la violencia patriarcal que atraviesa al capitalismo: un ejercicio que ya está siendo llevado a cabo teóricamente en los estudios, por ejemplo, de los feminicidios en México, tarea abordada por Rita Segato ${ }^{31}$. Además, la lucha contra la violencia sobre el cuerpo de las mujeres se está convirtiendo en un factor de movilización clave del movimiento feminista, tal y como muestra el movimiento Ni una menos en Latinoamérica, particularmente fuerte en territorios como Argentina. Dicho movimiento, en 2016, llevó incluso a este país a la convocatoria de una huelga feminista, que posteriormente ha tenido un fuerte impacto en las movilizaciones del 8 de Marzo en el territorio español ${ }^{32}$, y supone una articulación novedosa de la movilización política, pues el concepto de huelga no se restringe aquí al modelo clásico de la relación salarial.

La siguiente clave para la comprensión del presente tiene que ver con la conceptualización y articulación de alternativas posibles, que, en un momento en que $l o$ viejo no ha terminado de morir y lo nuevo no ha terminado de nacer, se convierte en una difícil empresa. La oposición al ALCA no es capaz de enseñarnos mucho al respecto, puesto que a pesar de una posición de confrontación común, no parecía tener una propuesta sustitutiva clara. En cualquier caso, las propias estrategias de resistencia que movimientos como Mesoamericanas por una vida digna ${ }^{33}$ han ido poniendo sobre la mesa, hace que nuestra atención pueda focalizarse en el refuerzo de las economías locales por un lado, y, por otro, la búsqueda de una integración regional alternativa que no pase por los intereses de agrupación basados en lógicas mercantiles. $\mathrm{La}$ primera de las propuestas permite una introducción de formas de vida económicas ajenas al mercado ya en el presente, ofreciendo soluciones concretas y apegadas al

$30 \quad$ Ibidem, p. 69. Asimismo, y para este último punto, cf. Ibidem pp. 66-73. Una interesante caracterización de la relación entre violencia y subjetivación en relación con los roles asignados a los géneros en el sistema capitalista puede encontrarse en N. Salobral "Transformaciones del trabajo desde una perspectiva feminista", en VV. AA. Economía feminista...pp. 95-110.

31 V. R.L. Segato, La guerra contra las mujeres. Madrid, Traficantes de Sueños, 2016.

32 V. a este respecto, [https://www.eldiario.es/internacional/Argentina-luto-calles-mujeres-asesinadas_0_571492847. $\mathrm{html}$, así como [http://hacialahuelgafeminista.org/manifiesto-8m/].

33 Cf. A. F. Torres R., "Las mujeres mesoamericanas resistiendo a las crisis", en VV. AA., Economía Feminista... pp. 29-46. En este artículo citado, es interesante hacer ver cómo las propias integrantes del colectivo entienden que algunas de sus propuestas de carácter económico están adecuadas al corto plazo. Sus textos, además, oponen una lógica de resistencia de la existencia muy interesante, en la que, tal y como comentan "muchas de las integrantes de las Mesoamericanas en Resistencia resisten al capitalismo neoliberal y heteropatriarcal desde sus iniciativas de generación de ingresos. Su resistencia: no dejarse morir como población desechable" (p. 38). La lucha contra la denominación como población desechable ha de relacionarse, sin duda alguna, con una época del capitalismo en que éste parece generar cada vez más "población superflua” (R. Kurz, "Unrentable Menschen. Ein Essay über den Zusammenhang von Modernisierungsgeschichte, Krise und neoliberalem Sozialdarwinismus", 2005, disponible en: [http://www.exit-online.org/link.php?tabelle=autoren\&posnr=237]), de manera evidente en el Norte Global. A partir de aquí, las experiencias latinoamericanas pueden servir de base para imaginar identidades de resistencia adecuadas en contextos occidentales. En otro orden de cuestiones, durante toda esta sección, cf. A. Pérez Orozco, Aprendizajes... pp. 74-79. 
territorio. Permiten no sólo una mejora inmediata de las condiciones de vida, sino un cuestionamiento de las propias dinámicas que la determinan en el capitalismo: la pregunta por la transformación de las formas de trabajo y cuidados se encuentran presentes en este tipo de alternativas. Asimismo, y al calor de una concepción de la transformación política al modo de una permacultura - es decir, aquel diseño agrícola y socioeconómico basado en los patrones del ecosistema natural- se pretende que la proliferación de alternativas permitan un ambiente favorable en el que sea posible realizar cambios aún más profundos. En este sentido, ha de favorecerse una propagación de estas propuestas, huyendo de su carácter marginal. Estrategias para ello son la relación y colaboración con el Estado si ésta se muestra deseable, y, en la medida de lo posible, la transferencia de estas proposiciones a contextos urbanos.

Ha de realizarse una salvedad de importancia. En estas propuestas, la utilización de ciertas nociones, como natural, o vida, puede que nos alerte, dado el riesgo de una posible caída en planteamientos esencialistas. En mi opinión, tal peligro es fácilmente expiable si los roles y las actividades, actitudes y características asociados a ellos se comprenden como algo contingente, contextualizado, y siempre abierto a la discusión de los participantes en el proceso.

Por lo que respecta a una integración regional alternativa, y ya para cerrar esta sección, ha de decirse que la oposición al ALCA se acompañó de una propuesta en que los gobiernos han jugado roles desiguales, y que ha cristalizado en propuestas de distinto corte (ALBA, UNASUR). Si bien a pesar de las reticencias que puedan tenerse, esta experiencias pueden servirnos en Europa para concebir modos de organización distintos que no pasen por el sistema de la UE - bien alternativos, bien complementarios a esta- y que, fundamentándose en la perspectiva crítica feminista, partan de una visión de la economía amplia. Asimismo, y dada la actual interconexión de la economía a nivel mundial, es necesario repensar espacios de articulación política supracontinentales, más allá de los existentes.

\section{La mirada feminista ante los tratados de Comercio e Inversión}

Tras el recorrido que hemos realizado, se ve con facilidad el modo en que las experiencias latinoamericanas nos ayudan a conformar una mirada feminista sobre los $\mathrm{TCI}^{34}$. El análisis ha de sostenerse sobre dos pilares fundamentales: en primer lugar, el posicionamiento de la vida en el centro, con atención al eje heteropatriarcal del capitalismo y su estructuración, atendiendo a las desigualdades que genera. Asimismo, la identificación del modelo sobre el que se asientan los tratados, comprendiéndose como un proceso que incide en la agudización del conflicto capital/vida, con reestructuraciones cambiantes en los elementos que lo constituyen (aumento de la violencia patriarcal como instrumento para su perpetuación, rearticulación de las cadenas globales de cuidados etc.).

Por otro lado, en orden a mejorar el análisis de los impactos hay que tener presente una perspectiva que no oponga a unos países frente a otros, sino que incida en la división pueblos/capitales. Al mismo tiempo, ha de hablarse en términos vitales que sean

34 En esta sección, A. Pérez Orozco "Qué implica mirar desde los feminismos a la hora de abordar los TCI". Esquema abierto al debate para pensar cómo abordar la nueva oleada de TCI, Mayo 2018. Utilizado con permiso de la autora. 
comprensibles para todxs, y utilizar la vida de las mujeres como un punto de vista privilegiado para la crítica. Esto determina nuestras herramientas de conocimiento, que han de usar metodologías tanto cuantitativas como cualitativas, evitando siempre el sobredimensionamiento de lo técnico. Por último, nuestros análisis han de prestar atención al corto y largo plazo. La mirada ha de estar puesta no sólo en el horizonte de la reducción de daños, sino en la construcción de alternativas factibles que permitan superar el actual modelo socioeconómico. En esta última tarea, hay ciertos horizontes que aparecen con claridad como algo deseable a alcanzar (soberanía alimentaria, energética), y otros que requieren todavía ser discutidos (concepciones del trabajo y cuidados, posible instauración de comunes como modo de articulación social, etc.). Aquí creemos que estas claves nos pueden ayudar a desmadejar el panorama que TCI como el TTIP o el ya aprobado CETA imponen en el contexto eurooccidental. Desde luego, el análisis habrá de adaptarse a un contexto en que hay que tener en cuenta factores que lo distinguen del latinoamericano, como podrían ser un mayor peso de los núcleos urbanos, así como la existencia de una moneda común, y la jerarquía que impone. Sea como fuere, es más lo que nos une que lo que nos diferencia.

Cabe destacar que la propuesta de Amaia Pérez Orozco no ha sido la única que, partiendo de un trabajo de reflexión sobre experiencias prácticas, ha desembocado en la construcción de herramientas teóricas ${ }^{35}$. En torno a este asunto nos gustaría mencionar siquiera brevemente la elaboración del Tratado Internacional de los Pueblos para el control de las empresas transnacionales ${ }^{36}$, un documento enmarcado en la campaña global Desmantelemos de las Corporaciones y pongamos fin a la impuni$\mathrm{dad}$, en que participan teóricos y movimientos sociales ${ }^{37}$. Esta propuesta, que incide en la arquitectura de la impunidad que antes hemos mencionado, intenta señalar los pilares de la transformación jurídica necesaria para el control efectivo del poder corporativo haciendo efectiva la necesidad de subordinar los beneficios empresariales a los derechos laborales y medioambientales, así como el desmantelamiento de las actuales asimetrías en la relación empresas/Estados-nación.

La descripción breve de nuestra situación y la mención de estas propuestas esperamos que haya posibilitado una perspectiva más amplia de nuestro presente, y algunas ideas de posibles horizontes de transformación que unan bajo una tarea común al Norte y Sur Global.

Sólo nos resta decir que, por sus características, quizá sea en la Academia donde con mayor frecuencia se impone el pesimismo de la razón que hemos mencionado. El remedio a este se da sólo en la difícil tarea de su disposición como una herramienta al servicio de la transformación, con toda la dificultad que esto supone. Ahora, sólo así podremos contagiarnos del optimismo contagioso de nuestros deseos de cambio, tan necesario.

35 Aunque sea evidente, el texto que hemos presentado aquí tampoco se trata del primer intento de reflexión acerca de las posibilidades de la Economía Feminista y la Economía Social y Solidaria como material para la construcción de alternativas, ni tampoco es la primera vez que las experiencias acaecidas en este continente son analizadas en vistas a la problematización de horizontes de transformación más allá del cortoplacismo imperante actualmente. A este respecto, valgan dos ejemplos sencillos: J. Hernández Zubizarreta, P. Ramiro "Empresas transnacionales en Latinoamérica: ¿hacia un nuevo modelo de desarrollo?, en Viento Sur, n 112, Octubre 2010, pp. 15-22, así como N. Quiroga Díaz, "Economías feminista, social y solidaria. Respuestas heterodoxas a la crisis de reproducción en América Latina”, en Iconos. Revista de Ciencias Sociales, n 33, Quito, enero 2009, pp. 77-89.

36 Cf. J. Hernández Zubizarreta, E. González y P. Ramiro, op. cit.

37 Cf. su página web en el siguiente enlace: [www. stopcorporateimpunity.org]. 
